\title{
CONCEPCIONES DE LOS ESTUDIANTES SOBRE LA CLASE ACADEMICA
}

\author{
Rolando Escorcia Caballero* \\ Alex Gutiérrez Moreno* \\ Roberto Figueroa Molina ${ }^{* * *}$
}

\begin{abstract}
RESUMEN: El presente texto explica las concepciones, creencias y pensamientos de los estudiantes en formación docente sobre la clase académica. Los estudiantes entienden la clase como un espacio para el trabajo académico en el que los profesores y los alumnos establecen relaciones interpersonales en un ambiente planeado intencionalmente para la formación. Además, entienden que la clase no es un constructo homogéneo, puesto que en ella se desarrollan acciones que conllevan a orientaciones simplistas del quehacer educativo o bien actividades que promueven el carácter multicausal, que implican los procesos y acciones del mundo de la clase.

Palabras claves: Clase académica. Creencias de los estudiantes. Categorías de pensamiento. Modelo ecológico. Modelo proceso-producto. Concepción ecléctica.
\end{abstract}

\section{THE STUDENTS' CONCEPTIONS ON ACADEMIC CLASSROOMS}

ABSTRACT: The present paper explains the conceptions, beliefs and thoughts on academic classrooms of students in teaching training. Those students understand classrooms as a space for academic

Magíster en Educación y profesor de la Facultad de Ciencias de la Educación, Universidad del Magdalena (Colombia). E-mail: rolando.escorcia@unimagdalena.edu.co

** Magíster en Educación y profesor de la Facultad de Ciencias de la Educación, Universidad del Magdalena (Colombia).E-mail: alexgutierrez66@hotmail.com

*** Doctor en Educación y profesor de la Facultad de Ciencias de la Educación, Universidad del Atlántico (Colombia). E-mail: robertofigueroamolina@gmail.com 
work in which teachers and students establish relationships in an environment intentionally established for training. Also, they understand that classrooms are not a homogeneous constructs, since both actions that entail simplistic orientations of teaching and educational activities that promote the multicausal character implying the processes of the classroom world are developed in it.

Key words: Academic class. Students' beliefs. Thought categories. Ecological model. Process-product model. Eclectic conception.

\section{Pensamiento del estudiante sobre el evento clase}

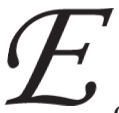

l pensamiento del estudiante para profesor sobre el ambiente académico. En un programa de formación para docentes, la visión del mundo con que los estudiantes inician deriva fundamentalmente de las experiencias educativas que vivieron. El avance en la carrera supone la estructuración de un pensamiento consecuente con la profesión docente, esto es, un pensamiento pedagógico, didáctico, sobre la escuela, la vida en el aula, entre otros. Sin embargo, si bien las concepciones de los estudiantes suponen cambios a través de su formación, éstas son más estables, cuanto más llevan formando parte del sistema de creencias de cada persona (Mellado, 1997).

Comprender las creencias y pensamientos de los estudiantes en formación docente y asumirlos como objeto de investigación resulta importante, entre otras razones, por lo que Glasersfeld (1988) ha llamado desilusión generalizada al analizar el estado actual de la enseñanza de las matemáticas, que puede atribuirse a la enseñanza de las ciencias en general. Desilusión que, según el autor, exige la revisión de teorías tradicionales de la educación y señala supuestos como: la concepción de enseñanza, del aprendizaje y de lo que es conocer; acciones que tienen ocurrencia en el espacio-tiempo denominado clase.

Acerca de la importancia de este tipo de estudio, Llinares (1992) señala que el acceder a los significados que los estudiantes para profesores y profesores en ejercicio atribuyen a los "objetos" de su trabajo debe ayudar a explicar tanto la efectividad del programa de formación, como a comprender por qué se comportan de determinada forma o generan determinados dilemas de enseñanza ante la acción de enseñar. 
En los últimos años la investigación sobre didáctica de las ciencias se ha consolidado en torno al estudio de las ideas de los alumnos como punto de partida de la enseñanza (Pozo et al., 1991), así como a sus ideas y significaciones previas en relación con los contenidos, especialmente en las ciencias experimentales. Para estos fines, se han comprometido muchos investigadores con trabajos de diferente orden y características, cuyos resultados apuntan a la tesis de que, para enseñar ciencias, es pertinente conocer las ideas que los alumnos tienen sobre ellas y desde ese presupuesto aplicar estrategias didácticas encaminadas a modificarlas de acuerdo con los propósitos de la educación, el contexto y los intereses del estudiante. Desde la psicología cognitiva, Shuell (1987) valora las ideas previas de los alumnos respecto del aprendizaje de nuevos conceptos; argumentos que han favorecido el avance investigativo del pensamiento del estudiante en la vida académica.

Por otro lado, el tema de las creencias lejos de ser un asunto marginal se constituye en uno central y de importancia destacada. Estas consideraciones han sido subrayadas, incluso, para la producción científica. Según Giraldo (2001), los conceptos científicos de una teoría, sus leyes y principios, las normas metodológicas de la investigación, los valores compartidos por un grupo de científicos, en fin, todo aquello que constituye una ideología específica conforma una intrincada red que es más compleja que la misma teoría. Sostiene además que los elementos de carácter no científico, pero que forman parte de la práctica real de la actividad científica, son tan responsables de encadenar la imaginación de los profesionales de una ciencia como los elementos científicos. Giraldo da un alto reconocimiento a las creencias, postulando, además, que son también parte esencial de las teorías científicas y, por lo mismo, del desarrollo de las ciencias. Para el autor, las creencias producen convicciones que la educación se encarga de legar a las nuevas generaciones, así como también la divulgación científica, constituyéndose en tradiciones y hasta verdades admitidas sin más.

Las creencias son marcos mediacionales desde las cuales se observa la realidad y orientan la conducta, siendo también válido para la experiencia académica de la clase. Ella está constituida por un conjunto de elementos y relaciones que determinan el mundo de la vida en aula. Sus actores fundamentales son los estudiantes y el profesor, quienes de 
diferente manera observan el mundo y poseen ideas distintas de la clase: qué se hace y cómo debe actuar en ésta. Cada uno posee convicciones acerca del rol que debe desempeñar cada quien en este evento. Por tanto, sus roles se desarrollan a partir de las orientaciones que sus convicciones dictan. El maestro observa su oficio con fundamentos científicos que orientan su quehacer, pero del mismo modo cuenta con creencias y esquemas de pensamiento fruto de su experiencia y de las tradiciones que al respecto existen, que definen su rol tanto o más que los basamentos científicos.

Para Koballa y Crawley (citados por Mellado, 1996) aunque el término concepción o creencia educativa se ha utilizado en investigación con distintos matices, la creencia o concepción implica una valoración sobre algo y, según Tobin, en ellas juega un importante papel la viabilidad, la componente social y la predisposición para actuar. La investigación sobre las concepciones, creencias y pensamiento del estudiante se enmarca en un campo recientemente iniciado en didáctica de las ciencias, el cual ha sido más abundante en relación con los maestros.

Según Gil (1991), subyace una concepción de la formación del profesorado que se sintetiza en las siguientes tesis:

1. Los profesores cuentan con ideas, comportamientos, actitudes... sobre la enseñanza/aprendizaje de las ciencias con los que es preciso conectar explícitamente en cualquier actividad de formación.

2. Un buen número de las creencias, comportamientos etc. sobre la enseñanza de las ciencias revelan una aceptación acrítica de lo que podríamos denominar una docencia de "sentido común", de "lo que siempre se ha hecho", que se convierte en obstáculo para una renovación de la enseñanza.

3. Sin embargo, si se facilita un trabajo colectivo de una cierta profundidad en torno a problemas de interés, los profesores podemos cuestionar las concepciones y prácticas asumidas acríticamente y construir conocimientos que son coherentes con los que la literatura especifica recoge como fruto de la investigación e innovación didáctica. 
Los estudiantes en formación docente, quienes actuarán como maestros de nuevas generaciones, presentan ideas, concepciones y actitudes sobre la enseñanza y aprendizaje de las ciencias (Mellado et al., 1999); así mismo, presentan ideas sobre el contexto en donde se dan estos procesos, la clase, que resultan de la idiosincrasia de la vida escolar a la cual han asistido. Estas ideas no siempre son modificadas, siendo algunas estables durante la carrera. Así entonces, a pesar del diplomado, los maestros mantienen ideas y creencias que se perpetúan en su práctica, "de forma que muchos maestros enseñan con métodos didácticos muy similares a los que ellos preferían cuando eran alumnos" (Huibregse et al., 1994, citado por Mellado, 1997). Por ello, es muy importante reconocer el pensamiento didáctico de los estudiantes para maestros, de suerte que sirva para una revisión crítica y constructiva acerca del papel del docente en la clase académica.

La investigación didáctica y, en particular, la formación de profesores, ha empezado a considerar el papel determinante de las ideas que mantienen los profesores y estudiantes para profesor, sobre lo que son los elementos que articulan sus actividades o son objeto de estudio durante el período de formación (instrucción, aprendizaje, objeto de la educación, materias curriculares) (Llinares, 1990, 1992). Para Llinares (1992), la importancia que tienen las ideas de los profesores en formación radica en que "filtran" la información que proceden de las situaciones concretas y fundamentan posibles acciones en contextos todavía no presentes.

Estas ideas previas de los profesores abarcan desde el conocimiento y creencias del contenido pedagógico en relación con las metas, objetivos y propósitos educativos, su papel como profesores y los niños como aprendices, pasando por el conocimiento y creencias sobre el contenido particular de las materias (Bromme y Brophy, 1986; citados por Llinares, 1992).

Los resultados de los procesos educativos se dan en función de las relaciones que los sujetos tienen en la dinámica académica y, en buena medida, al compromiso que adquieren a partir del dictado de sus pensamientos, concepciones, percepciones y significados sobre la vida académica. El trabajo didáctico del maestro y el proceso de formación del estudiante en el mundo institucionalizado ocurren preferencialmente en el espacio-tiempo llamado clase, entendiendo que éste 
corresponde a la formalidad institucional y/o al encuentro del docente y los estudiantes en cualquier espacio, o a través de la red en los "campus virtuales de aprendizaje".

En el microcontexto de la clase, la enseñanza y el aprendizaje son mediados por la vivencia del docente y del estudiante, de sus experiencias y expectativas, de tal manera que el reconocimiento del pensamiento, el contexto vivencial y cultural del estudiante como ejes articuladores del hecho educativo permitirá incluir nuevos elementos en la dinámica didáctica a partir de la realidad del educando.

El mundo y las cosas en él se revelan a los individuos como distintos elementos y estímulos. Canter, Brown y Groat (1985) han sostenido que muchos psicólogos han enfatizado que la habilidad para desenvolverse en el mundo se relaciona muy de cerca con la habilidad para formar categorías y construir sistemas de clasificación, en los cuales un conjunto de estímulos no idénticos se tratan de manera equivalente. Estos autores se apoyan en los planteamientos de Smith y Medin, quienes argumentan que si tuviéramos que relacionarnos con objetos, eventos, comportamientos o sentimientos, asumiendo que cada objeto o situación es único, entonces el esfuerzo que habría que hacer en la vida inteligente sería virtualmente imposible. De allí que el comportamiento del estudiante en el mundo del aula se enmarca en las categorías que ellos usan y los conceptos que asignan a tales categorías, que en el presente estudio se relacionan con el evento clase.

Para De Zubiría y De Zubiría, las categorías son complejos instrumentos del conocimiento caracterizadas porque las proposiciones que la arman poseen una estructura, un orden, unas interrelaciones. No son un conjunto ni un amontonamiento, según ocurre con los conceptos. Es un sistema jerárquico organizado de proposiciones mediante inclusiones, subcontinencias, sobrecontinencias y nexos laterales (véase la figura 1).

Ontoria (1995), basándose en la teoría cognitiva del aprendizaje, de Ausubel, plantea que la ordenación jerárquica también puede mostrar el conjunto de relaciones entre un concepto y aquellos otros subordinados a él, surgiendo la diferenciación entre los conceptos al mostrar interrelaciones conceptuales especificas. Sobre la base de la formación de complejas redes conceptuales que constituyen las categorías en el sistema de pensamiento, el individuo interpreta y actúa en el 
mundo. En nuestra comprensión, asociamos detalles y realizamos inferencias: estos son elementos constituyentes del proceso total de comprensión; pero nunca percibimos un gesto o una acción en sí misma, los percibimos como parte de una estructura más compleja, como un patrón o red de relaciones que está allí afuera, que tiene un impacto más directo que el atribuible a la inferencia y a la empatía, y que no puede deducirse ni reconstituirse a partir de un montón de datos (Martínez, 1991).

\section{Figura 1}

Estructura de una categoría

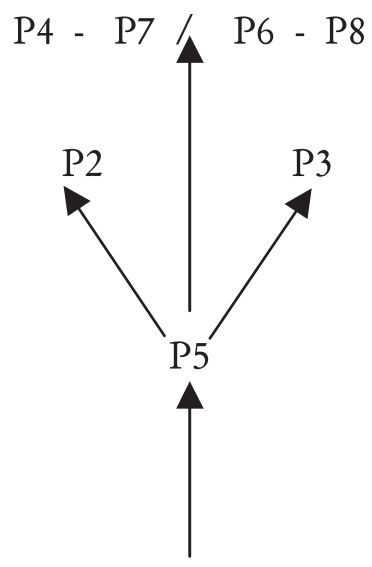

P1

Fuente: De Zubiría y De Zubiría, 1992.

Las categorías presentan un carácter interpretativo del mundo, con ellas se producen los discursos pedagógicos y se sustentan las prácticas cotidianas en el aula de clases. Cuando sus contenidos conceptuales no han sido suficientemente reflexionados a la luz de la ciencia pedagógica, muchas veces están dominados por las experiencias idiosincrásicas de los maestros y actúan como obstáculos para la innovación y avance 
de su quehacer didáctico, ofreciendo resistencias conscientes o inconscientes.

Es importante estudiar los sistemas conceptuales del pensamiento sobre los procesos didácticos en la experiencia de la clase de los estudiantes en formación docente, en atención a que ellos poseen un cúmulo de significados producto de su interacción con el medio y de los años de escolaridad que han tenido; la intervención educativa debe entonces partir de las posibilidades de razonamiento y aprendizaje que caracterizan al estudiante, implicando partir de su nivel de competencia cognitiva y del pensamiento didáctico que ha construido en su devenir.

Se pretende, a través de la comprensión del evento clase, tener algunas herramientas conceptuales que sirvan para reflexionar acerca del trabajo didáctico. Así mismo, comprender cómo conciben y entienden los estudiantes los aspectos relacionados con el hecho educativo es una contribución para el desarrollo de la educación y el saber pedagógico, así como al conocimiento del pensamiento de los aspectos académicos de los estudiantes, permitiendo reconocer su visión, así como su percepción de la vida en el aula y el sentido que le asigna.

Planteamiento del problema y metodología de investigación

Existe en la vida académica una vivencia cotidiana, la clase, que remite el interés de los estudiosos de la educación. Esta experiencia, estudiada en sus diferentes aspectos y desde distintos enfoques, constituye un objeto digno de ser conocido para fundamentar tanto la teoría como la práctica didáctica. Teniendo en cuenta que hasta ahora las investigaciones han tendido a reconocer los procesos didácticos, las relaciones, el aprendizaje, las influencias internas y externas de su éxito o fracaso, las concepciones y creencias sobre la ciencia, la enseñanza y el aprendizaje de los docentes y han dejado de lado las propias concepciones, creencias y pensamientos del estudiante sobre la clase, la presente investigación da cuenta del pensamiento en cuanto a las categorizaciones conceptuales del estudiante sobre la clase.

Plantear esta problemática toma sentido por cuanto la mayoría de los procesos educativos están enmarcados en esta experiencia específica de actividad académica; además, en el entendido que el abordaje 
de esta experiencia conlleva la puesta en escena de las expectativas y experiencias previas que orientan el comportamiento dentro de ella, resulta importante reconocer cuáles categorías conceptuales son del dominio de los sujetos que a este evento asisten, especialmente, si están formándose como educadores y bien pronto, deberán movilizar comportamientos de otros, en sus propias experiencias de dirección de clases.

El modo como el estudiante visualiza el hecho educativo ha sido poco considerado; de hecho, el docente le asigna poca importancia, a pesar que tenerlo presente constituye una herramienta didáctica para la construcción y planificación curricular. Comprender cómo categorizan un grupo de sujetos el evento clase es un aporte al reconocimiento de la problemática que surge en relación con el pensamiento, las concepciones y creencias del estudiante en tanto permitirá identificar las categorías con que los estudiantes en formación docente entienden la clase académica.

El presente estudio de caso es de carácter descriptivo e interpretativo. Por su propia naturaleza no pretende legitimar generalizaciones, sino que, utilizando los criterios de fiabilidad y validez de la investigación cualitativa (Goetz y Lecompte, 1988; Marcelo y Parrilla, 1991), contribuir a ampliar, precisar y dinamizar la comprensión del fenómeno objeto de estudio.

La investigación se apoyó en las siguientes técnicas: composición escrita, Clasificación Múltiple de Ítems (CMI) y entrevista de clasificaciones estructuradas, en relación con los mismos ítems en términos de frecuencia con que se presentan en sus clases y de importancia que le asignan a cada ítem.

El proyecto se desarrolló inicialmente con 32 estudiantes de los distintos programas de la Facultad de Ciencias de la Educación de la Universidad del Magdalena, quienes realizaron la composición escrita. Esta consiste en un ensayo libre propuesto a partir de la pregunta orientadora: ¿Qué entiende por clase académica? En esta técnica el estudiante plantea desde sus propias convicciones y experiencias lo que a su juicio es la clase académica. Esta información es pertinente para considerar los elementos que desde el propio pensamiento de los estudiantes constituyen el evento clase, siendo de singular importancia en la construcción del instrumento para la CMI. 
Construcción de los ítems para la CMI

La información recolectada en las composiciones fue analizada en sus contenidos (análisis de contenidos) para la elaboración de los preitems (tarjetas con las proposiciones acerca de la clase), demandando para el análisis un proceso de codificación. En la investigación cualitativa, la codificación es un modo sistemático de desarrollar y refinar las interpretaciones de los datos (Taylor y Bogdan, 1990) que consiste en transformar en forma sistemática los datos "brutos" (como "ordinariamente" los plantean los sujetos) y presentarlos agregados en unidades que permiten una descripción precisa de las características pertinentes del contenido (Bardín, 1986).

Se ha utilizado el término unidad de sentido o unidad temática para significar aquellos tópicos de los protocolos que representan elementos constituyentes de la clase. La composición de los sujetos es dividida así en unidades de sentido con los cuales finalmente se describe un preitem; es decir, una proposición que representa un elemento de la clase en concordancia con los contenidos.

A partir del análisis se construyeron los preítems (40). Estos fueron enviados a un grupo de tres expertos, junto con una guía de valoración para considerar pertinencia, claridad y neutralidad. Sus recomendaciones permitieron refinar algunos ítems y la eliminación de otros.

Con los ítems resultantes se realizó una prueba piloto de la CMI con ocho estudiantes de los 32 participantes. Luego se analizaron aquellos ítems que presentaban problemas para ser clasificados en algún grupo y aquellos que, a juicio de los estudiantes, presentaban dificultades en la lectura o interpretación. Esto permitió eliminar algunos preítems, quedando 24 ítems para la aplicación final de CMI (véase la tabla 1).

Clasificación Múltiple de Ítems (CMI): clasificaciones libres y dirigidas (Canter, Brown y Groat, 1985; Pacheco, 1986: Páramo, 1998-1999). La técnica CMI consiste en una entrevista en la cual a los participantes le son presentados ítems o enunciados (elementos de la clase) en tarjetas diseñadas para tal fin, las cuales deben clasificar según su propio criterio. 


\section{Tabla 1}

Ítems definitivos para la entrevista CMI

\begin{tabular}{cl}
\hline No & \multicolumn{1}{c}{ ÍTEMS } \\
\hline 1 & La construcción de conocimiento en clase \\
2 & El compartir conocimiento en la clase \\
3 & La actividad académica en cualquier parte \\
4 & Las relaciones interpersonales en la clase \\
5 & La guía del maestro en la clase \\
6 & La exposición de conocimiento en clase \\
7 & El desarrollo de temas por maestros y estudiantes en clase \\
8 & La aclaración de dudas en clase \\
9 & El ambiente académico de diálogo en la clase \\
10 & El análisis de saberes en clase \\
11 & La planeación metodológica de la clase \\
12 & La formación en valores \\
13 & La expresión de pensamientos en clase \\
14 & La orientación y motivación a la investigación \\
15 & Las actividades dinámicas y creativas en la clase \\
16 & El aprendizaje a partir de experiencias \\
17 & El desarrollo de autoaprendizaje en clase \\
18 & Los procesos de evaluación en clase \\
19 & Los estados de ánimo en clase \\
20 & Los debates para fortalecer el conocimiento en clase \\
21 & El uso de recursos didácticos en clase \\
22 & El empleo de recursos bibliográficos en clase \\
23 & El espacio para la reflexión \\
24 & El interés por aprender y participar en clase \\
\hline &
\end{tabular}

\section{Aplicación de la CMI definitiva}

En la entrevista se pidió a los participantes que clasificaran las tarjetas de acuerdo con criterios propios, de tal forma que cada grupo formado tenga algo en particular que los hace similar, debiendo 
argumentar las razones de su clasificación. Se tomaron notas en formatos de entrevistas, teniendo en cuenta los números que las tarjetas presentan en su respaldo. El entrevistado formó tantos grupos como pudo y, cuando manifestaron no querer o poder hacer más clasificaciones, se consideraron suficientes los sorteos y se terminó la entrevista.

Los resultados de las clasificaciones realizadas se organizaron en tablas que contenían un número de identificación, el número de sorteos, el grupo de tarjetas, el criterio con los cuales los clasificó y el número de identificación del ítem (véase la tabla 2).

\section{Tabla 2}

Clasificaciones libres de los estudiantes

\begin{tabular}{|c|c|c|c|}
\hline 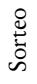 & 号 & Criterios & Tarjetas \\
\hline \multirow{7}{*}{1} & 1 & Interacción docente alumno & $1-2-7-9$ \\
\hline & 2 & Factores que influyen para un buen aprendizaje en clases & $5-6-8-13-14-20$ \\
\hline & 3 & Uso de los diferentes tipos de evaluación & $10-18-23$ \\
\hline & 4 & Diferentes maneras de aprender & $16-17-24$ \\
\hline & 5 & Hombre un ser social & $4-12-19$ \\
\hline & 6 & Aula de clases viva & 3-11 \\
\hline & 7 & Buenos recursos, excelente aprendizaje & $15-21-22$ \\
\hline \multirow{5}{*}{2} & 1 & Responsabilidades del docente en clases & $5-8-12-14$ \\
\hline & 2 & Técnicas para transmitir conocimiento & $15-21-22$ \\
\hline & 3 & Retroalimentación del conocimiento & $1-2-6-7-10-13-16-20-23$ \\
\hline & 4 & Ambiente Educativo ideal & 4-9-17-19-24 \\
\hline & 5 & Factores que conllevan a la investigación & $3-11-18$ \\
\hline \multirow{3}{*}{1} & 1 & Recursos didácticos & $3-5-6-7-15-16-20$ \\
\hline & 2 & Motivación por parte del docente & 4-9-11-12-14-18-23- \\
\hline & 3 & Interés del estudiante en el desarrollo de la clase & 1-2-8-10-13-17-19-21-22-24- \\
\hline \multirow{3}{*}{2} & 1 & Importancia del trabajo en grupo & 1-2-4-6-8-9-10--11-12-13-16-20-24 \\
\hline & 2 & Motivación a la investigación & 7-14-21-22-23- \\
\hline & 3 & Diferentes maneras que emplea el docente para evaluar & $3-5-15-17-18-19$ \\
\hline
\end{tabular}

Estos resultados son procesados mediante un Análisis de Escalogramas Multidimensionales (MSA, por su sigla en inglés). Los datos derivados de las clasificaciones libres son procesados por el software Phychometric Análysis Package (PAP). 
"Aunque el MSA (Lingoes, 1968) es solamente uno de un número de técnicas de escala multidimensional, tiene unas propiedades especiales que lo hacen ideal para comprender las relaciones de los datos cualitativos" (Wilson, 1995, p. 263). En particular, una de las ventajas de estos procedimientos es que permiten la representación visual de la estructura de los datos en un gráfico total, en un diagrama de puntos que resume las relaciones entre los ítems. El gráfico representa las relaciones anotadas en las clasificaciones; es decir, las relaciones empíricas.

El MSA (Shye, 1978) se utiliza para analizar perfiles, los cuales se representan en el espacio de tal forma que, para cada ítem, existe una ubicación. Para efecto de la investigación que se describe, este hecho es relevante, pues la contigüidad de los puntos (ítems, elementos de la clase) está relacionada con la categoría de respuestas de los ítems. Partiendo de esta información, los datos pueden ser analizados, comparando los sistemas de clasificación entre los participantes del estudio, entre los elementos o ítems sorteados, o entre los conceptos y categorías a las cuales se asignan los elementos y brinda la posibilidad de construir teoría a partir del conjunto complejo de relaciones en la estructura de los datos (Canter, Brown y Groat, 1985; Páramo, 19981999; Pacheco, 1996).

De acuerdo con Shye, Elizur y Hoffman (1994), es extremadamente útil representar conceptos multivariados como espacios físicos con una extensión. La analogía del espacio de conceptos puede ser formalizada como un mapeo del conjunto (típicamente infinito) de ítems que pertenecen al concepto en un espacio geométrico. La contigüidad representa las relaciones empíricas entre los ítems y, según Shye, Elizur y Hoffman (1994), para todo ítem observado se asigna un punto en el espacio geométrico y, una vez establecida la noción de proximidad o distancia entre los ítems, ésta también debe ser representada en el espacio de conceptos $\mathrm{y}$, de acuerdo con ello, el investigador determina la naturaleza de la proximidad conceptual con la cual él o ella está relacionada.

En consecuencia, cualquier clasificación conceptual de ítems se representa por una partición apropiada del espacio en regiones. Frecuentemente, tales regiones geométricas (subconjunto del espacio) corresponden a subconceptos bien formulados de los conceptos. Este principio es utilizado en la presente investigación para determinar con cuales categorías los estudiantes entienden el evento clase académica. 


\section{Resultados}

La clase es entendida como un espacio de trabajo académico cuyos protagonistas principales - maestro y estudiantes - establecen relaciones interpersonales en un ambiente planeado intencionalmente para la formación, no exclusivamente en relación con el conocimiento, que muchas veces resulta ser más predominante, sino también en relación con el mundo social de los valores y principios de convivencia. Este espacio es formalizado institucionalmente y ello constituye frecuentemente una de las mayores dificultades en el entendimiento y puesta en escena de la experiencia académica, puesto que en ese marco se condensan normas verticales que impiden el despliegue de las expectativas de los estudiantes e incluso de los docentes. No obstante, la conceptualización de la clase no es homogénea, ya que se pueden observar tendencias que van desde una visión reduccionista, hasta una mucho más compleja que implica un conjunto sistemático de acciones, temáticas y procesos que trascienden hasta el plano socioafectivo y motivacional.

En este orden de ideas, la clase es vista desde cuatro categorías: una socioafectiva (ítems 4,12,17, 19 y 24) que le da relevancia a los aspectos sociales, motivacionales y axiológicos; otra categoría relacionada con los diferentes procesos desarrollados alrededor del conocimiento (ítems $1,2,6,7,8,10$, y 13). La tercera categoría comprende la dimensión didáctica, vista por los estudiantes como el conjunto da $a c$ ciones académicas (ítems 5, 11, 15, 18, 20, 21 y 22) o actividades diseñadas para el logro de los objetivos académicos, y por último, la conceptual, (ítems 3, 9, 14, 16 y 23), con la cual representan las visiones más globales de los aspectos de la clase.

A lo largo del análisis de las categorías en las clasificaciones libres, son relievados dos modelos que aparecen en los planteamientos de los estudiantes de manera ecléctica. Estos dos modelos son: proceso producto y ecológico. Estos, sin embargo, no son explicitados en las entrevistas, sino que subyacen en ellas. En ese sentido, el trabajo comprensivo consistió en derivar de la información los fundamentos categoriales que caracterizan el pensamiento de los estudiantes objeto de estudio.

En cada categoría, es posible identificar una serie de procesos que definen los roles que desempeñan cada uno de los actores: maestros y 
alumnos. En este sentido, es significativo reflexionar acerca de dos aspectos: a) la importancia que le dan al docente en el proceso de enseñanza y en la dinámica de la clase, apreciándose una marcada tendencia a una concepción centrada en la asignatura, donde se privilegia los contenidos y b) una concepción enmarcada en una perspectiva de orientación social, que podemos acercar al modelo ecológico, donde observan el desarrollo de la actividad académica como un proceso complejo multifactorial, en donde debe primar el aprendizaje a partir de experiencias en un ambiente de diálogo, reflexión análisis y construcción de conocimiento.

El primer aspecto de reflexión se corresponde con la concepción de clase fundamentada en el modelo proceso producto, allí el docente es considerado como un dador de información o, en ciertos casos, como el que explica cierto concepto para que después sea repetido por el estudiante en evaluaciones diseñadas para tal fin, que privilegian el aprendizaje memorístico y repetitivo (en el marco de este modelo la evaluación siempre se refiere a un acto del docente y no se plantea como parte de la cotidianidad académica, permanente y autoadministrada; por tanto, no aparecen conceptos como autoevaluación o coevaluación). Desde esta perspectiva, el docente es considerado como vertical y desmotivante, con lo cual no contribuye a un buen aprendizaje de los alumnos, quienes han dejado ver una relación entre docente motivante y buen aprendizaje. Esta implica el repertorio de actividades diseñadas para el desarrollo de la clase, bajo la premisa que según sean planeadas estratégicamente dichas actividades, teniendo en cuenta los recursos didácticos y bibliográficos, se obtienen resultados satisfactorios en la motivación, participación y aprendizaje de los estudiantes. En este sentido, se advierte la responsabilidad por parte del docente en el planeamiento del trabajo académico y los estudiantes se ven como beneficiarios pasivos del dictado de la información. No se plantea así motivación intrínseca de los estudiantes, sino más bien motivación extrínseca.

El segundo aspecto de reflexión concierne al modelo ecológico, que a la luz del análisis corresponde al deber ser y no al ser, es decir, a la percepción de los estudiantes de cómo debe ser y desarrollarse el acto académico para obtener un mejor aprendizaje. Entienden la vida en el aula como una red viva de intercambios socioculturales. En ésta dinámica, el intercambio se entiende como un devenir en el cual los estudiantes y 
el docente ejercen roles diferentes de intercambio, creación y transformación de significados, en donde se debe propender por la comprensión de los conceptos, más que memorizarlos y repetirlos, en ocasiones sin siquiera asimilarlos. Aquí el dinamismo y la creatividad del docente constituyen elementos fundamentales para un buen aprendizaje, así como el estímulo hacia el análisis y la comprensión de los conceptos.

En este aspecto, el docente se constituye en alguien que guía, aclara dudas, comparte conocimiento y orienta y motiva al estudiante. Le da especial significado al papel que debe jugar como alumno y al conjunto de acciones tendientes a su formación integral, partiendo de su accionar hacia el aprendizaje, colocando en el mismo plano el conjunto de acciones académicas que le permiten un mayor crecimiento conceptual, con la participación del docente, pero cuestionando su papel transmisionista y percibiéndolo como un orientador de procesos que diseña y planifica acciones que conlleven al debate y fortalecimiento del conocimiento en oposición al papel del maestro tradicionalista, expositivo y poco creativo. Por un lado, conciben los debates como actividades de metodologías interactivas, por tanto dinámicas, que permiten fortalecer el conocimiento, y al maestro, lo conciben como el que debe guiar, orientar, pero no siempre desde una perspectiva rígida o sobre todo expositiva o transmisionista, quien debe organizar y desarrollar actividades creativas y dinámicas, que en últimas coadyuvarían a un buen aprendizaje de los estudiantes.

Un elemento encontrado que contribuye con un mejor aprendizaje, indistintamente del modelo bajo el cual miran la clase, lo constituye el lugar donde se desarrolla la actividad académica. Los estudiantes consideran que no debe estar circunscrita al aula de clases, sino que puede y debe desarrollarse en espacios diferentes, los cuales de alguna manera motivan su interés por aprender y facilitan el autoaprendizaje.

Por otro lado, se entiende que existe una correlación positiva entre los aprendizajes en función de los estados de ánimo. Es precisamente en este donde se explicita con mayor claridad las concepciones del modelo ecológico de la comprensión de la vida en el aula. Los estudiantes le asignan al aspecto socioafectivo un papel muy importante, considerándolo como un factor significativo en los procesos de enseñanza y de aprendizaje. Se concibe la clase no solamente desde la transmisión de información, sino que se encuentra una tendencia bien 
marcada a valorar el conjunto de relaciones socioafectivas que de hecho se presentan en la clase, pero que al parecer, los docentes no le dan la suficiente importancia. La marcada dependencia entre los aprendizajes y los factores sociales y motivacionales, en función de dichas relaciones, más que en el saber acerca de los contenidos, es un buen punto de partida para la reflexión didáctica y la búsqueda del mejoramiento de la docencia y para el planeamiento curricular. Entre otras cosas, porque la formación axiológica que ha ganado discursivamente muchos defensores depende más del mundo social del aula que de todo intento por argumentar expositivamente los principios y normas de convivencia.

Lo anterior supone también una concepción epistemológica frente al conocimiento, relacionado con las ideas que presentan sobre la clase, la enseñanza y el aprendizaje, cuyo fundamento se enmarca en el constructivismo. Allí el conocimiento no se tiene como algo absoluto o acabado, al que acceden en la clase como descubrimiento de realidades develadas, sino más bien, aquel es el resultado de la actividad protagonizada por el estudiante en interacción con el profesor y demás estudiantes de un curso dado. Es decir, para los estudiantes el conocimiento constituye un saber que, del mundo susceptible de ser construido y no como algo acabado, dado externamente y al cual se puede acceder de alguna manera. Desde la perspectiva cooperativa, la clase se constituye en un espacio-tiempo de diálogo constructivo sobre el saber: diálogo de saberes, donde es válido la expresión de pensamientos y conocimientos relativos a un tema determinado sujeto de análisis.

Los estudiantes se entienden como protagonistas de su aprendizaje, sujetos de creación y transformación de sus significados el resultado del ambiente de aprendizaje generado por el docente, pero sobre todo, del conjunto de vivencias que tienen lugar en la clase. El sujeto del aprendizaje es entendido como un procesador de información de los elementos de la realidad que, en función de su estructura cognitiva, asigna valoración a tales elementos, de acuerdo a su idiosincrasia. Crea en la medida que deconstruye y reconstruye su estructura de significados. Esto explica porqué la realidad es aprehendida de distinto modo por un grupo de alumnos en un mismo escenario y un mismo docente, ya que cada uno re-crea significados y la creación de los nuevos significados conlleva cambios o transformaciones de la estructura de significado existente. 


\section{Conclusiones}

Los estudiantes entienden la clase como un espacio para el trabajo académico, en el que profesores y alumnos establecen relaciones interpersonales en un ambiente planeado intencionalmente para la formación. Los fines de dicha relación no son exclusivamente para la adquisición o construcción de conocimientos, que en el medio educativo parece tener mayor preponderancia, sino también en relación con el mundo social de los valores y principios de convivencia. En ese sentido se le da un carácter institucional, marco que se constituye en el contexto normatizado que determina el camino curricular del desarrollo académico. Por esta circunstancia, es visto como un momento ritualizado con una estructura de poder centrada en el profesor, no solo en términos de disciplina conductual, sino también en relación con el conocimiento que se asemeja al modelo transmisionista, rutina que impide el despliegue de las expectativas de los estudiantes e incluso de los docentes.

La conceptualización de la clase no es un constructo homogéneo en los estudiantes, puesto observan orientaciones con una visión simplista del quehacer educativo o bien orientaciones que admiten el carácter multicausal, por tanto, complejo de la vida del mundo de la clase, que implica un conjunto sistemático de acciones, temáticas y procesos. Los modelos proceso producto y ecológico sirven de marco para ubicar este eclecticismo conceptual de la clase.

En el pensamiento del estudiante coexisten y se relacionan cuatro categorías: a) categoría aspectos socioafectivos, cuyos elementos constitutivos definen los aspectos sociales, motivacionales y axiológicos; b) categoría conocimiento, donde se manifiestan los caracteres teleológicos dados con mayor frecuencia al evento clase, es decir, existe el supuesto que el conocimiento en sí constituye el fin de las actividades académicas, donado por el profesor al alumno que ejemplifica al modelo transmisionista, no obstante que las expectativas frente a la clase por parte de los estudiantes para maestro tienden a un modelo constructivista; c) categoría acciones académicas, que define actividades diseñadas para el logro de los objetivos académicos, comprendiendo los elementos y relaciones de la dimensión didáctica de la clase, y d) Categoría conceptual, con la cual representan las visiones más globales de los aspectos de la clase. 
En cada una de las categorías, los estudiantes definen roles del profesor y de los estudiantes. El pensamiento categorial de los estudiantes se organiza, entonces, a partir de lo que creen es el papel de cada actor en el proceso académico. Aunque los estudiantes para maestros le dan gran importancia a las actividades del alumno en clase parece ser que este hecho no tiene mayor ocurrencia en sus experiencias. Esto, en principio, demuestra el predominio de una didáctica centrada en el profesor como transmisor de conocimiento, con una lógica del poder que desequilibra la balanza en favor del docente.

La vida en el aula es comprendida como una red de intercambios, los cuales no se limitan a la relación maestro alumno, sino también convoca los intercambios entre los estudiantes. La importancia de concebir las vivencias del aula como redes de intercambios radica en que se construye comunidad y se puede poner en evidencia que el conocimiento es un producto social, que se configura de acuerdo a los intereses sociales y no son asuntos absolutos a los cuales hay que hacer reverencias. Además, puede cuestionarse la premisa de que el alumno aprende lo que enseña el profesor, pues en las relaciones de intercambios entre iguales se aprenden o se fortalecen los saberes, a través de los debates y el diálogo de saberes. Aquí el dinamismo y la creatividad del docente constituyen elementos fundamentales para fomentar aprendizajes deseables, así como el estímulo hacia el análisis y la comprensión de los conceptos.

Al docente se le adjudica el cometido de guiador, que propicia la aclaración de dudas, comparte conocimiento, orientando y motivando al estudiante.

Los estudiantes le otorgan mucha importancia a los aspectos socioafectivos durante el evento clase. Establecen una relación directamente proporcional entre el avance del aprendizaje y los estados de ánimo que se viven en la clase. La motivación, a su vez, la relacionan con la propuesta metodológica del docente, en el sentido que si estas son creativas y dinámicas favorecen la motivación y participación del alumno Se puede observar en sus consideraciones que la motivación es más extrínseca que intrínseca. De cualquier forma, los estados de ánimo son considerados como un factor significativo en los procesos de enseñanza y de aprendizaje, que se ven limitados en un modelo de transmisión de información, al igual que otros elementos considerados 
como positivos. Por otro lado, la formación axiológica está ligada al conjunto de vivencias en el aula, en especial al tipo y calidad de relaciones socioafectivas que a la exposición o dictados de normas y principios.

Existe una perspectiva epistemológica frente al conocimiento, la enseñanza y el aprendizaje, que se aproxima al constructivismo. El conocimiento es entendido como resultado del esfuerzo constructivo del alumno y no como descubrimiento, no como una verdad revelada por un depositario de la verdad. Desde la perspectiva cooperativa, la clase se constituye en un espacio-tiempo de diálogo constructivo sobre el saber: diálogo de saberes, donde es válido la expresión de pensamientos y conocimientos relativos a un tema determinado.

A pesar de las preferencias de los estudiantes por modelos transversales, la experiencia actual parece conducirse por caminos diferentes. Las rutinas cotidianas de los estudiantes parecen estar fundamentando el establecimiento de esquemas de trabajo académicos que no permiten un mayor desarrollo profesional docente, en la medida en que no se favorece la reflexión sobre el saber qué y cómo del quehacer pedagógico.

La evaluación aunque se presenta con mucha ocurrencia en la clase y también le es dada considerable importancia, es concebida como una actividad del docente y no como una acción propia del estudiante. Esta heteronomía frente a la evaluación puede derivarse del modelo transmisionista al cual están expuestos.

Recebido em novembro de 2006 e aprovado em outubro de 2008.

\section{Referencias}

AUSUBEL, D. et al. Psicología educativa: un punto de vista cognoscitivo. México: Trillas, 1983.

BARDIN, L. Análisis de contenido. Madrid: Akal, 1986.

CANTER, D.; BROWN, J.; GROAT, L. A multiple sorting procedure studding conceptual systems. In: Brenner, M.; Brown, J.; CANTER, D. The research interview: uses and approaches. London: Academic, 1985. 
COLAS, M.P. Enfoques en la metodología cualitativa: sus prácticas de investigación. In: Buendia Eisman, L.; Colas Bravo, P.; Hernandez Pina, F. Métodos de investigación en psicopedagogía. Madrid: McGraw Hill, 1998.

DE ZUBIRIA, M.; DE ZUBIRIA, J. Biografía del pensamiento. Bogotá: Mesa Redonda-Magisterio, 1992.

GIL, D. ¿Qué hemos de saber y saber hacer los profesores de ciencias?: intento de síntesis de las aportaciones de la investigación didáctica. Enseñanza de las Ciencias, Madrid, v. 9, n. 1, p. 67-77, 1991.

GLASERSFELD, E. Learning as constructive activity. In: GLASERSFELD, E. The contruction of knowledge: contribution to conceptual semantics. Salinas, Ca.: Intersystem, 1987. (GLASERSFELD, E. Constructivismo: el paradigma, el aprendizaje, la enseñanza y el cambio conceptual. Trad. de Félix Bustos. Santiago: Univ. Santiago de Cali, 1993).

GOETZ J.P.; LECOMPTE, M.D. Etnografía y diseño cualitativo en investigación cualitativa. Madrid: Morata, 1988.

LLINARES, S. El conocimiento y las creencias de los profesores de matemáticas y la innovación educativa. Investigación en la Escuela, Sevilla, n. 11, p. 61-69, 1990.

LLINARES, S. Los mapas cognitivos como instrumento para investigar las creencias epistemológicas de los profesores. In: MARCELO, C. La investigación sobre formación del profesorado: métodos de investigación cualitativa. Argentina: Cincel, 1992. (compilación).

MARCELO, C. La investigación sobre formación del profesorado: métodos de investigación y análisis de datos. Argentina: Cincel, 1992

MARCELO, C. Calidad y eficiencia de los profesores: una educación con calidad y equidad. Madrid: OEI, 1999.

MARCELO, C.; PARRILLA, A. El estudio de caso: una estrategia para la formación del profesorado y la investigación didáctica. In: Marcelo, C. et al. (Ed.). El estudio de caso en la formación del profesorado. Santiago: Tórculo, 1991. 
MARTÍNEZ, M. Investigación cualitativa etnográfica en educación. Caracas: Texto, 1991.

MELlADO, V. Concepciones y practicas de aula de profesores de ciencias en formación inicial de primaria y secundaria. Enseñanza de las Ciencias, Madrid, v. 14, n. 3, p. 298-302, 1996.

MELLADO, V.; GONZÁLEZ, T.; DIAZ-PINTO, M. Estudio longitudinal de las concepciones de estudiantes de maestro de educación primaria sobre la enseñanza de las ciencias. Campo Abierto, Badajoz, n. 16, p. 57-77, 1999.

MELLADO, V.; RUIZ, C.; BLANCO, L. Aprender a enseñar ciencias experimentales en la formación inicial de maestros. Bordón, Madrid, v. 49, n. 3, p. 273-278, 1997.

ONTORIA, A. Mapas conceptuales: una técnica para aprender. Madrid: Narcea, 1995.

PACHECO, J. La clasificación múltiple de ítems y el análisis de escalogramas multidimensionales. Suma Psicológica, Bogotá, v. 3, n. 1, p. 2537, 1996.

PÁRAMO, P. La ciudad como lugar. Bogotá: UPN, 1998.

PÁRAMO, P. La clasificación múltiple de items como soporte al trabajo investigativo en didáctica universitaria y de los proceso de construcción social del conocimiento en el aula. Bogotá: UPN, 1999.

POZO, J.A. et al. Las ideas de los alumnos sobre la ciencia: una interpretación desde la psicología cognitiva. Enseñanza de las Ciencias, Madrid, v. 9, n. 1, p. 83-94, 1991.

SHUELL, T. Cognitive psychology and conceptual change: implication for teaching science. Science Education, London, v. 71, n. 2, p. 237-250, 1987.

SHULMAN, L. Paradigmas y programas de investigación en el estudio de la enseñanza: una perspectiva contemporánea. In: Wittrock, M. (Comp.). La investigación de la enseñanza 1. Barcelona: Paidós, 1989. 
SHYE, S. Theory constructions and data analysis in the behavioral sciences. San Francisco: Jossey-Bass, 1978.

SHYE, S.; ELIZUR, D.; HOFFMAN, M. Introduction to facet theory. London: Sage, 1994.

TAYLOR S.J.; BOGDAN, R. Introducción a los métodos cualitativos de investigación: la búsqueda de significados. Buenos Aires: Paidós, 1990.

WILSON, M. Structuring qualitative data: multidimensional scalogram analys. In: WiLSON, M. et al. Research methods in psychology. London: Sage. 1995. 